\title{
Relationship between Para-aminohippurate Secretion and Cellular Morphology in Rabbit Proximal Tubules
}

\author{
Philip B. Woodhall, C. Craig Tisher, Charles A. Simonton, and \\ Roscoe R. Robinson, Division of Nephrology, Department of Medicine, \\ and the Department of Pathology, Duke University Medical Center, \\ Durham, North Carolina 27710
}

A B S T RACT Previous studies in the mammalian proximal tubule have suggested that para-aminohippurate (PAH) secretion is $\sim$ threefold greater in the straight segment, or pars recta, than in the convoluted segment, or pars convoluta. However, the possibility that the site of maximal PAH secretion might be related better to particular tubule segments as identified by cell type had not been explored. In addition, the presence or absence of differences in $\mathrm{PAH}$ secretion between morphologically identical regions of superficial (SF) vs. juxtamedullary (JM) proximal tubules has not been examined. These issues were studied using a combination of histologic methods and measurement of $\left[{ }^{3} \mathrm{H}\right] \mathrm{PAH}$ secretion in isolated perfused tubules. Measurements of microdissected SF and JM proximal tubules from young and adult rabbits revealed that SF proximal tubules were slightly but significantly longer than JM tubules ([young rabbits: $\mathrm{SF}, 8.69 \pm$ SE $0.14 \mathrm{~mm}$ vs. JM, $7.97 \pm \mathrm{SE} 0.13 \mathrm{~mm} ; P$ $<0.01$ ] [adult rabbits: SF, $10.61 \pm \mathrm{SE} 0.28 \mathrm{~mm}$; JM, $9.17 \pm \mathrm{SE} 0.19 \mathrm{~mm} ; P<0.001])$. Light and electron microscopy revealed three sequential segments $\left(S_{1}\right.$, $S_{2}$, and $S_{3}$ ) along the length of $S F$ and $J M$ proximal tubules as defined by cell type. PAH secretion was measured in each of these three segments by the isolated perfused tubule technique. Net PAH secretion in $\mathrm{fmol} / \mathrm{mm}$ per $\min$ in $\mathrm{SF}$ proximal tubules was: $\mathrm{S}_{1}, 281 \pm \mathrm{SE} \mathrm{21;} \mathrm{S}_{2}, 1,508 \pm \mathrm{SE} 104 ; \mathrm{S}_{3}, 318 \pm \mathrm{SE} 46$. Corresponding values in $\mathrm{JM}$ proximal tubules were $353 \pm \mathrm{SE} 31,1,391 \pm \mathrm{SE} 72$, and $188 \pm \mathrm{SE} 23$. Net PAH

This report was presented in part before the Southern Section of the American Federation for Clinical Research, New Orleans, La., 22 January 1976, and the combined session of the American Federation for Clinical Research and the American Society for Clinical Investigation, Atlantic City, N. J., 2 May 1976.

Dr. Woodhall was the recipient of a fellowship from the North Carolina Heart Association.

Received for publication 11 July 1977 and in revised form 5 December 1977. secretion did not differ between comparable segments of SF and JM proximal tubules. It is concluded that differences in $\mathrm{PAH}$ secretion along the proximal tubule correlate best with cell type rather than the arbitrary division of the proximal tubule into pars convoluta and pars recta according to its external configuration. Evidence of functional heterogeneity between comparable segments of $\mathrm{SF}$ and $\mathrm{JM}$ proximal tubules was not observed.

\section{INTRODUCTION}

It is widely held that secretion of the organic acid, paraaminohippurate, occurs principally in the straight portion, or pars recta, of the mammalian proximal tubule, whereas the contribution of the convoluted portion, or pars convoluta, to this active transport process is relatively minor. Tune, et al. (1) were the first to directly measure the secretion of $\left[{ }^{3} \mathrm{H}\right] p$-aminohippurate in isolated perfused segments of rabbit proximal tubule and found that the rate of secretion was approximately three times greater in the straight as compared to the convoluted segment. However, the division of the mammalian proximal tubule into two regions, the pars convoluta and the pars recta, defined by certain gross and external macroscopic features, runs counter to other morphologic data. Light, fluorescence, and electron microscopic observations, particularly those of Suzuki (2), Sjöstrand (3), Maunsbach (4), Rhodin (5) and Tisher, et al. (6) have revealed the presence of at least three morphologically distinct segments in all mammalian proximal tubules thus far studied in detail. Moreover, the distribution of the three major segments $\left(S_{1}, S_{2}\right.$, and $\left.S_{3}\right)$ defined according to cell type, does not conform to the division of the proximal tubule into a convoluted and a straight portion. For instance, in the rat (4), $S_{1}$ is limited to the beginning of the pars convoluta, $S_{2}$ includes the more distal portions of the pars convoluta and the beginning of the descending part of the 
proximal tubule, or pars recta, and $\mathrm{S}_{3}$ encompasses the remainder of the pars recta. Despite the extensive use of the rabbit kidney in the isolated perfused tubule procedure (7), it has not yet been established whether similar segmentation defined according to cellular morphology also exists in the rabbit proximal tubule.

The purpose of the present investigation was threefold. First, the rabbit proximal tubule was examined morphologically to establish the presence or absence of cellular segmentation. Second, after demonstration of the presence of three morphologically distinct segments in this species as well, para-aminohippurate $(\mathrm{PAH})^{1}$ secretion was measured in each segment by the isolated perfused tubule technique to determine whether the site or sites of maximal secretion of this organic acid have any relationship to cell type. Finally, to determine whether heterogeneity existed with respect to PAH secretion in comparable regions of proximal tubules from different nephron populations, secretory rates in each of the three cellular segments were measured in both superficial and juxtamedullary nephrons.

\section{METHODS}

\section{Anatomic studies}

Microdissection. Proximal tubules from superficial (SF) and juxtamedullary (JM) nephrons were dissected to establish the lengths of the convoluted and straight segments of the tubule. Five young, female, New Zealand white rabbits (mean age, 82 days; mean body weight, $2.1 \mathrm{~kg}$ ) and four adult female rabbits of the same species (mean age, 163 days; mean body weight, $3.5 \mathrm{~kg}$ ) were lightly anesthetized with i.v. pentobarbital sodium $(20-30 \mathrm{mg} / \mathrm{kg}$ body weight). $2 \mathrm{ml}$ of $10 \%$ (wt/vol) sodium ferrocyanide solution was then injected i.v. and $20 \mathrm{~s}$ after completion of the injection the left or right kidney was removed. Sagittal slices of the excised kidney were snap-frozen in liquid nitrogen and incubated overnight in a cold $\left(-18^{\circ} \mathrm{C}\right)$ solution of ethanol (95 vol\%) and concentrated hydrochloric acid (5 vol\%) containing $50 \mathrm{~g} / 100 \mathrm{ml}$ of ferric chloride. The kidney slices were then digested in $20 \%$ (wt/vol) hydrochloric acid at $45^{\circ} \mathrm{C}$ for $20-40 \mathrm{~min}$, at which time individual tubules could be freely dissected.

The SF proximal tubules were defined as those with at least one convolution on the kidney surface. The JM proximal tubules were defined as those belonging to a nephron whose glomerulus was situated immediately adjacent to the corticomedullary junction. Only tubules in which the glomerulus and the thin descending limb were attached were included in the study. Approximately $10 \mathrm{SF}$ and $10 \mathrm{JM}$ proximal tubules were removed from one kidney of each rabbit and transferred from the dissecting dish to a standard glass microscope slide. The exact transition from the convoluted to the straight portion of the tubule was marked on the slide. The tubule was then uncoiled gently, keep-

${ }^{1}$ Abbreviations used in this paper: JM, juxtamedullary; PAH, para-aminohippurate, $p$-aminohippuric acid; PC, pars convoluta; PR, pars recta; SF, superficial. ing the transition point stationary. The slide was then placed in a camera lucida (Wild Heerbrugg, Switzerland) and a tracing made of the tubule at $40 \times$. With a calibrated planimeter, the length of the entire tubule and its convoluted and straight segments were determined from the tracing.

Light and electron microscopy. Tissue for microscopic examination was obtained from three, healthy, young, female, New Zealand white rabbits. These animals were anesthetized lightly with pentobarbital sodium (20-30) $\mathrm{mg} / \mathrm{kg}$ body weight) before intravascular perfusion fixation of the left kidney was performed with Karnovsky's fixative diluted to one-half strength (osmolality: $960 \mathrm{mosmol} / \mathrm{kg} \mathrm{H}_{2} \mathrm{O}$ ) as previously described $(8,9)$. Thin coronal slices of these kidneys were immersed in the same fixative at room temperature for 4 additional $h$, then stored in a potassium phosphate buffer (osmolality: $950 \mathrm{mosmol} / \mathrm{kg} \mathrm{H}_{2} \mathrm{O}$ ).

Portions of both $\mathrm{SF}$ and $\mathrm{JM}$ proximal tubules were identified and chosen for microscopic examination. To examine early proximal convoluted tubules, blocks of tissue were selected from either the JM or outer cortical regions of the kidney slices and routinely processed and sectioned for light and electron microscopy (8, 9). Early proximal convoluted tubules were so identified because they were visibly connected to the renal corpuscle of their respective nephrons. Late proximal convoluted tubules were identified in one of several ways. A kidney slice was placed flat in a Petri dish containing buffer and directly visualized under a stereomicroscope at $60-150 \times$. The open lumina of wellpreserved straight portions of $\mathrm{SF}$ and JM proximal tubules were clearly visible. With standard micropuncture techniques, latex (General Biological Supply House, Chicago, Ill.) was injected into each individual tubule until the late convolution was filled in a retrograde manner. Blocks of tissue containing late SF or JM proximal convoluted tubules filled with latex were cut and processed for microscopy (10). Alternatively, late convolutions of SF and JM proximal tubules could occasionally be dissected directly from fixed tissue without the aid of acid digestion. These tubule fragments were also examined by microscopy. Manual dissection of straight portions of proximal tubules of SF and JM nephrons was also accomplished from the fixed tissue. These tubules usually contained either the final portion of the late proximal convolution at one end or the thin descending limb at the opposite end. Occasionally, the entire segment from the last convolution to the thin limb could be obtained. These individual straight segments of proximal tubules were divided into sequential $1-\mathrm{mm}$ lengths and processed for light and electron microscopy. With a combination of these histologic techniques, an anatomic profile of the entire proximal tubule was obtained.

\section{Physiologic studies}

Transport of PAH. After completion of the anatomic studies, the capacity of histologically defined segments of SF and JM proximal tubules to secrete PAH was determined by the isolated perfused tubule technique. General methods for the isolation and perfusion of tubular segments were those of Burg et al. (7). Animals utilized in the study were female New Zealand white rabbits similar in age and size to the younger animals used for the anatomic studies. Tubule segments selected for study were anatomically defined and chosen in the following manner. In SF nephrons, early convoluted segments $\left(S_{1}\right)$ were those either with the glomerulus attached or located more than $2 \mathrm{~mm}$ proximal to the beginning of the descending portion of the tubule. Late con- 
voluted segments $\left(S_{2}\right)$ were the $1-1.5 \mathrm{~mm}$ of tubule immediately before the start of the descending portion of the proximal tubule. Upper straight portions $\left(S_{2}\right)$ came from the outer one-half of the cortex, and lower straight portions $\left(S_{3}\right)$ consisted of the tubular segment starting at the corticomedullary junction and ending with the transition into the descending thin limb of Henle's loop.

Proximal tubules of JM nephrons were located either by their attachment to a JM glomerulus or to a long, thin limb descending into the inner medulla. Early segments of JM proximal tubule $\left(S_{1}\right)$ were selected by their attachment to the glomerulus or their position greater than $3 \mathrm{~mm}$ proximal to the beginning of the descending portion of the convolution (see Results for comment on the configuration of the JM proximal tubule). Late segments of JM convoluted tubule $\left(S_{2}\right)$ were taken from the $2-3 \mathrm{~mm}$ of tubule located just proximal to the straight portion. The straight portion $\left(\mathrm{S}_{3}\right)$ was that segment between the last convolution and the start of the descending thin limb. This segment was rarely greater than $1.5 \mathrm{~mm}$ in length and lay almost entirely within the outer medullary region.

Each segment of tubule was perfused with an ultrafiltrate of rabbit serum prepared by pressure dialysis at $4^{\circ} \mathrm{C}$ with an XM-50 membrane (Amicon Corp., Lexington, Mass.) and was bathed in $2 \mathrm{ml}$ of isosmotic rabbit serum from the same serum lot. Perfusate and bath were preequilibrated by bubbling with a $95 \% \mathrm{O}_{2}-5 \% \mathrm{CO}_{2}$ gas mixture for $10-20 \mathrm{~min}$ just before use. During the experiment the bath chamber was bubbled continuously with the same gas mixture and maintained at $37^{\circ} \mathrm{C}$.

The secretion studies in all segments of proximal tubule were performed in an identical manner. After the tubule was connected to perfusing and collecting pipettes, an initial equilibration period of $15 \mathrm{~min}$ in a bath of normal rabbit serum was allowed. The bathing fluid was then changed to rabbit serum containing $\left[{ }^{3} \mathrm{H}\right] \mathrm{PAH}$ (sp act, $130 \mathrm{mCi} / \mathrm{mmol}$, New England Nuclear, Boston, Mass.) at a chemical concentration of $24 \mu \mathrm{M}$. After a second 15-min equilibration period, three consecutive timed sample collections were made under oil using a constant volume pipette. In most experiments ouabain $(50 \mu \mathrm{M})$ was added to the bath after completion of the third collection and $5 \mathrm{~min}$ later a final timed collection was obtained. The perfusion rate ranged from 4-15 $\mathrm{nl} / \mathrm{min}$ in different experiments and was usually varied during separate collection periods of the same experiment by changing the height of a fluid column that was hydraulically attached to the perfusion pipette.

Secretion of PAH was also examined in the same manner in an additional group of JM and $\mathrm{SF} \mathrm{S}_{3}$ segments bathed and perfused with a slightly hypertonic ultrafiltrate of rabbit serum (osmolality: $350-360$ mosmol/ $\mathrm{kg} \mathrm{H}_{2} \mathrm{O}$ ) to simulate the more hypertonic environment of tubules in the outer medulla. The osmolality was increased by the addition of $\mathrm{NaCl}$.

To rule out the presence of leakage due to mechanical damage, $\left.{ }^{125} \mathrm{I}\right]$ iothalamate (Abbott Laboratories, N. Chicago, Ill.) or $\left[{ }^{14} \mathrm{C}\right]$ inulin (New England Nuclear) was added to the bath along with $\left[{ }^{3} \mathrm{H}\right] \mathrm{PAH}$. During each experimental period the bath was sampled to assure that the chemical concentration of PAH remained constant during each experiment and was similar in all experimental groups. Radioactivity in all samples was measured by liquid scintillation counting in Aquasol (American Cyanamid Co., Pearl River, N. Y.) plus $4 \%$ water by volume using a dual-channel scintillation spectrophotometer (Isocap 300, Searle Analytic Inc., Des Plaines, Ill.) or by gamma counting (Beckman Biogamma, Beckman Instruments, Inc., Fullerton, Calif.).
The quantity of PAH present in each sample was calculated from the observed counts, the counting efficiency for appropriate $\left[{ }^{3} \mathrm{H}\right] \mathrm{PAH}$ standard solutions, and the specific activity of the $\left[{ }^{3} \mathrm{H}\right] \mathrm{PAH}$.

Statistical comparisons were made with unpaired $t$ test analysis.

\section{RESULTS}

\section{Anatomy}

Gross observations obtained by microdissection of individual tubules. The term pars convoluta (PC) was applied to any portion of the proximal tubule that was visibly convoluted, and the term pars recta (PR), or straight portion of the proximal tubule, was used specifically to designate that portion which was visibly straight, or approximately so. The characteristic external configuration of dissected SF and JM proximal tubules is shown in Fig. $1 a$ and $b$. As indicated, there was a marked disparity between the configuration of these two types of tubules as observed under the dissecting microscope. Table I contains the measurements of dissected proximal tubules from both young and adult animals. The length of each segment for individual animals represents the mean value for the length of that segment in 9-12 tubules from the same rabbit. In younger animals the average total length of the SF proximal tubule $(8.69 \pm \mathrm{SE} 0.14) \mathrm{ex}-$ ceeded that of the JM proximal tubule $(7.97 \pm$ SE 0.13 ; $P<0.01)$. The absolute length of the PC of JM proximal tubules greatly exceeded that of $\mathrm{SF}$ proximal tubules $(P<0.001)$, whereas the converse was true for the PR of JM proximal tubules $(P<0.001)$. The $\mathrm{PC}$ of $\mathrm{SF}$ nephrons represented $\sim 60 \%$ of the total length of the proximal tubule, and the PC represented $\sim 90 \%$ of the total length of JM nephrons. In the adult group the relative lengths of the PC and PR segments in the two subpopulations of nephrons were nearly identical to those observed in younger animals. Again, in adult rabbits, the average mean length of the SF proximal tubule $(10.61 \pm \mathrm{SE} 0.28)$ exceeded that of the JM proximal tubule $(9.17 \pm \mathrm{SE} 0.19 ; P<0.001)$. In JM nephrons the straight, or PR segment, represented only the terminal $1 \mathrm{~mm}$ or less of the tubule. All segments of the proximal tubule were longer in the adult animals except the straight portion of JM proximal tubules, which remained essentially unchanged (young, $0.81 \pm$ SE 0.05 vs. adult, $0.84 \pm 0.06 ; P, \mathrm{NS}$ ).

Morphological observations obtained with light and electron microscopy. Light and transmission electron microscopy were employed to define the cellular composition of $\mathrm{SF}$ and JM proximal tubules. It was found that the proximal tubule of both SF and JM nephrons could be divided into three distinct regions as previously demonstrated in the rat $(3,4)$, mouse (5), and 


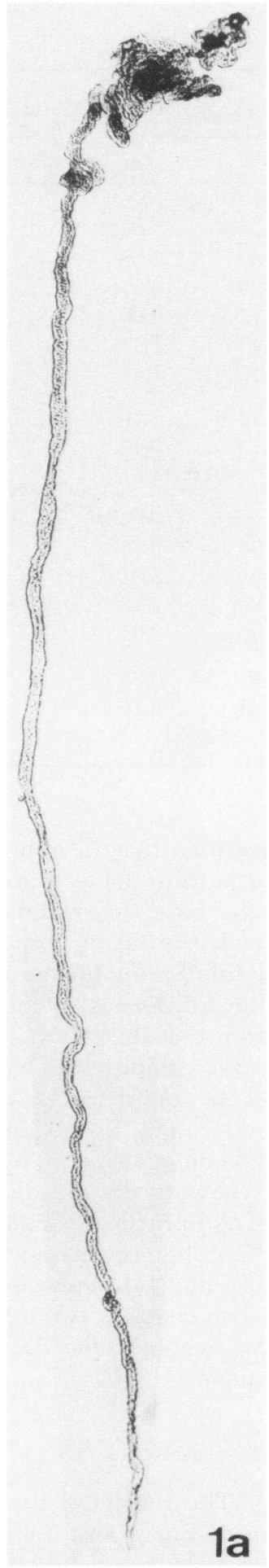

Figure la Photomicrograph illustrating the gross appearance of a microdissected proximal tubule from a $S F$ nephron. Magnification, $\times 42$. rhesus monkey (6). The segments will be referred to as $S_{1}, S_{2}$, and $S_{3}$ to conform with terminology currently in wide usage to describe this region of the nephron. Each of the three segments was identified according to the appearance of a particularly distinct and uniform cell type within each segment. Cellular architecture in each segment was comparable in $\mathrm{SF}$ and JM nephrons. The $S_{1}$ segment, which represented the early proximal convoluted tubule in all nephrons, began after a connecting or "neck" region of variable length of up to $90 \mu \mathrm{m}$ as described by Sperber (11) and Sch $\phi$ nheyder and Maunsbach (12). Cells in the $S_{1}$ region were columnar with a tall, thick brush border, numerous elongated mitochondria frequently contained within invaginations of the basal plasmalemma, and an apical region which contained a well-developed endocytic apparatus (Fig. 2a). The basement membrane in the $S_{1}$ region was thicker than that of other segments. In SF nephrons, cells typical of the $S_{1}$ segment were never located in late proximal convolutions or the PR, nor were they seen in the late convolutions just preceding the short straight terminal portion of JM proximal tubules. However, the exact point of transition from $S_{1}$ to $S_{2}$ was not identified with certainty.

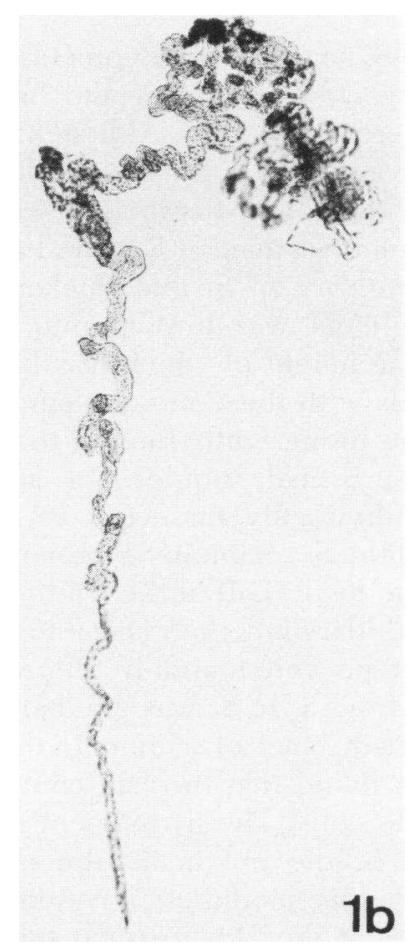

Figure $1 b$ Photomicrograph illustrating the gross appearance of a microdissected proximal tubule from a JM nephron. Magnification, $\times 42$. 
TABLE I

Results of Microdissection in Kidneys of Young and Adult Rabbits

\begin{tabular}{|c|c|c|c|c|c|c|c|c|c|}
\hline \multirow[b]{2}{*}{ Group } & \multirow[b]{2}{*}{ Age } & \multirow{2}{*}{$\begin{array}{c}\text { Animal } \\
\text { wt }\end{array}$} & \multirow{2}{*}{$\begin{array}{l}\text { Kidney } \\
\text { wt }\end{array}$} & \multicolumn{2}{|c|}{$\begin{array}{l}\text { Total length of } \\
\text { proximal tubule }\end{array}$} & \multicolumn{2}{|c|}{ Length of PC } & \multicolumn{2}{|c|}{ Length of PR } \\
\hline & & & & SF & $\mathrm{JM}$ & SF & JM & SF & $\mathrm{JM}$ \\
\hline & days & $\mathrm{kg}$ & $\mathrm{g}$ & \multicolumn{2}{|c|}{$m m$} & \multicolumn{2}{|c|}{$m m$} & \multicolumn{2}{|c|}{$m m$} \\
\hline Young animals & & & & $(n=48)$ & $(n=47)$ & $(n=50)$ & $(n=47)$ & $(n=49)$ & $(n=47)$ \\
\hline 1 & 80 & 1.8 & 9.55 & 7.50 & 7.87 & 4.66 & 7.55 & 2.94 & 0.32 \\
\hline 2 & 93 & 2.2 & 8.25 & 8.85 & 7.15 & 5.29 & 6.37 & 3.56 & 0.79 \\
\hline 3 & 71 & 1.9 & 5.60 & 8.21 & 7.37 & 5.12 & 6.60 & 3.09 & 0.75 \\
\hline 4 & 79 & 2.0 & 5.95 & 8.83 & 8.45 & 5.45 & 7.47 & 3.38 & 0.97 \\
\hline 5 & 89 & 2.6 & 8.54 & 9.74 & 8.94 & 5.93 & 7.91 & 3.81 & 1.03 \\
\hline Mean & 82 & 2.1 & 7.58 & 8.69 & 7.97 & 5.30 & 7.16 & 3.36 & 0.81 \\
\hline SE & & & & 0.14 & 0.13 & 0.10 & 0.13 & 0.08 & 0.05 \\
\hline$P$ & & & & \multicolumn{2}{|c|}{$<0.01$} & \multicolumn{2}{|c|}{$<0.001$} & \multicolumn{2}{|c|}{$<0.001$} \\
\hline Adult animals & & & & $(n=42)$ & $(n=43)$ & $(n=42)$ & $(n=46)$ & $(n=42)$ & $(n=44)$ \\
\hline 1 & 165 & 4.0 & 11.6 & 12.88 & 10.87 & 8.06 & 9.70 & 4.60 & 1.16 \\
\hline 2 & 160 & 3.2 & 7.1 & 8.50 & 8.86 & 5.01 & 8.01 & 3.48 & 0.86 \\
\hline 3 & 161 & 3.3 & 8.6 & 9.88 & 8.72 & 5.83 & 7.78 & 4.05 & 0.94 \\
\hline 4 & 166 & 3.5 & 9.4 & 11.32 & 8.41 & 7.00 & 8.01 & 4.32 & 0.40 \\
\hline Mean & 163 & 3.5 & 9.2 & 10.61 & 9.17 & 6.47 & 8.35 & 4.11 & 0.84 \\
\hline $\mathrm{SE}$ & & & & 0.28 & 0.19 & 0.23 & 0.16 & 0.09 & 0.06 \\
\hline$P$ & & & & \multicolumn{2}{|c|}{$<0.001$} & \multicolumn{2}{|c|}{$<0.001$} & \multicolumn{2}{|c|}{$<0.001$} \\
\hline
\end{tabular}

Cells of the $S_{2}$ segment of the proximal tubule were tall, cuboidal to low, columnar and had a relatively short brush border (Fig. 2b). Although mitochondrial profiles were numerous, they were less prominent than in the $S_{1}$ segment. Microbodies were more common in this segment than in $S_{1}$. The basement membrane was thinner than $S_{1}$, but thicker than $S_{3}$. The basal plasmalemma was thrown into numerous invaginations, the height of which was less than in the $S_{1}$ region. Cells with these morphological characteristics formed the lining epithelium of the late convolutions of SF proximal tubules. In addition, serial sections of individually dissected PR revealed that cells typical of the $S_{2}$ region in SF proximal tubules extended into the lower half of the PR to the vicinity of the corticomedullary junction. Distal to this transition, cells of the $S_{3}$ type were found. In SF proximal tubules the transition from $S_{2}$ to $S_{3}$ was gradual and appeared to extend over a distance of $\sim 1 \mathrm{~mm}$. In the JM proximal tubules it was found that the late convolutions were composed of $S_{2}$ cells. The $S_{2}$ segment ended $\sim 1 \mathrm{~mm}$ from the end of the proximal tubule, again in the vicinity of the corticomedullary junction. The terminal portion, or PR, of the JM proximal tubule, although not as straight as that of SF nephrons, was composed entirely of $S_{3}$ rather than $S_{2}$ cells.

The $S_{3}$ cell (Fig. $2 c$ ) was cuboidal and had a longer brush border than the $S_{2}$ cells, although not quite as long as that described in the rat (4). The cell height was distinctly less than that in either $S_{1}$ or $S_{2}$. Profiles of mitochondria were not as profuse as in $S_{2}$, and invaginations of the basal plasmalemma were infrequent. Sections of tissue taken perpendicular to the path of proximal tubules in the outer stripe of the outer medulla allowed dozens of tubules to be surveyed and indicated that the terminal segment of all proximal tubules was composed of $S_{3}$ cells. Thus, all $S_{1}$ and $S_{2}$ cells were found in the cortex, while $S_{3}$ cells were confined chiefly to the innermost regions of the cortex and the outer stripe of the outer medulla. Fig. 3 indicates schematically the distribution of the three segments of the proximal tubule in SF and JM nephrons. The PC is heterogeneous with respect to cell type in both SF and JM nephrons. The PR of SF proximal tubules also contains two cellularly discrete regions, $\left(S_{2}\right.$ and $\left.S_{3}\right)$, whereas, the PR of JM nephrons contains $S_{3}$ cells alone.

\section{Physiologic observations}

PAH secretion. The results of the PAH secretion studies are shown in Fig. 4 and Table II. Fig. 4 depicts the results of a characteristic group of measurements performed in $S_{1}$ segments dissected from five SF proximal tubules. The net secretory rate remained relatively constant with time and it was not altered by varying the perfusion rate over a range of 4-15 $\mathrm{nl} / \mathrm{min}$. The addition of ouabain at a concentration of 

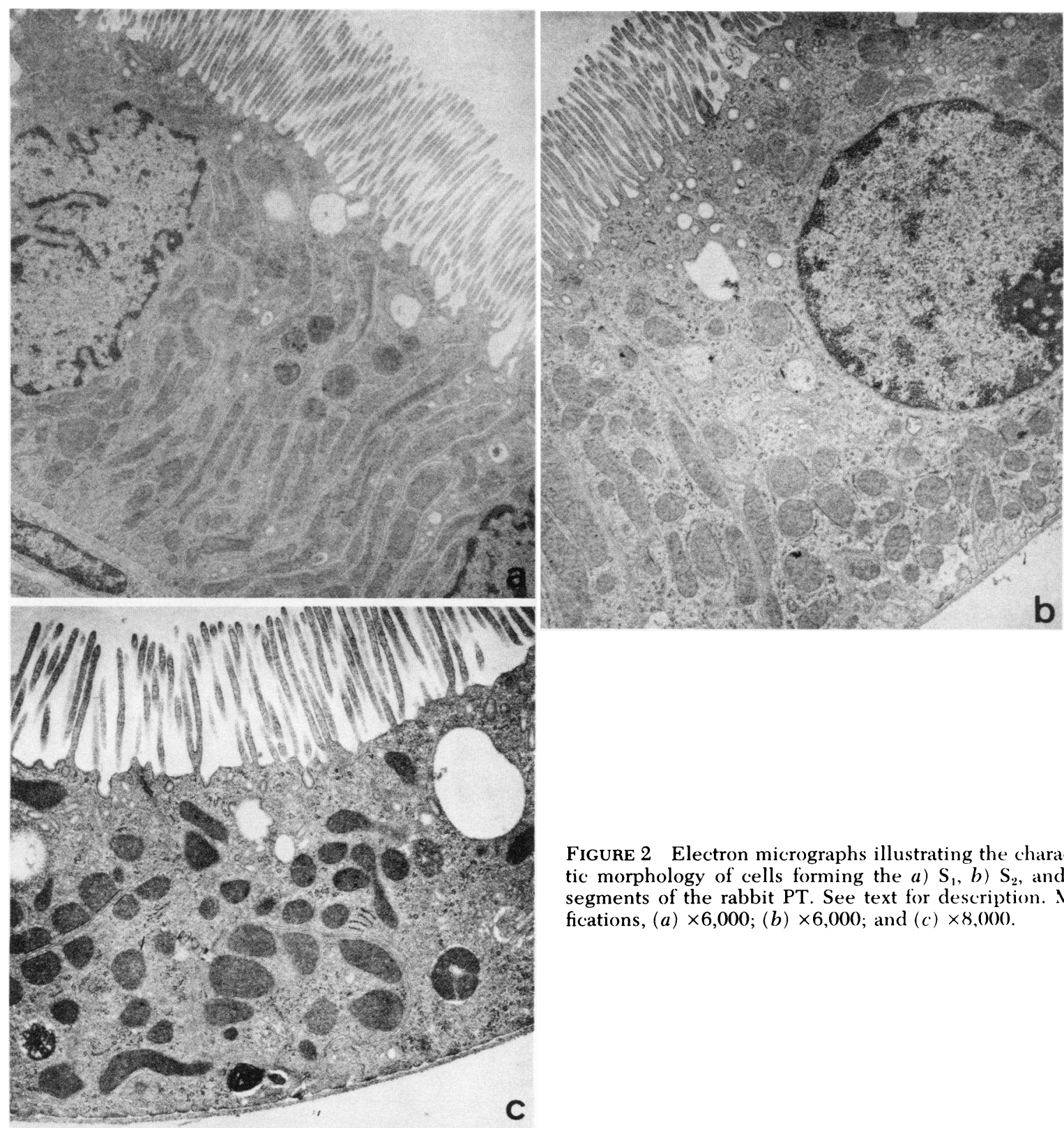

FIGURE 2 Electron micrographs illustrating the characteristic morphology of cells forming the $\left.a) S_{1}, b\right) S_{2}$, and c) $S_{3}$ segments of the rabbit PT. See text for description. Magnifications, $(a) \times 6,000 ;(b) \times 6,000$; and $(c) \times 8,0(0)$.

$50 \mu \mathrm{M}$ markedly reduced net secretion within minutes. Net PAH secretory rates for each of the three histologically defined segments of the proximal tubule in both SF and JM nephrons are listed in Table II and the results are summarized in Fig. 5. Maximum PAH secretion was limited strictly to the $S_{2}$ segments of both SF and JM proximal tubules. In SF proximal tubules, the maximum net secretion of PAH was not confined solely to the straight portion of the $\mathrm{S}_{2}$ segment, inasmuch as three of the eight $S_{2}$ segments examined were entirely convoluted in character and exhibited a mean secretory rate of $\sim 1,500 \mathrm{fmol} / \mathrm{mm}$

per min, a value which corresponded quite closely to that observed in five straight $S_{2}$ segments $(1,350$ $\mathrm{fmol} / \mathrm{mm}$ per $\mathrm{min})$. In both $\mathrm{SF}$ and JM nephrons, the $\mathrm{S}_{3}$ segment possessed the lowest net secretory rate, although the difference in net $\mathrm{PAH}$ secretion between the $S_{1}$ and $S_{3}$ segments in $S F$ nephrons was not statistically significant $(P>0.30)$. However, a small but statistically significant difference in mean secretory rates was observed between $S_{1}$ and $S_{3}$ segments of JM nephrons $(P<0.025)$. This difference was largely due to the inclusion of one tubule from a JM nephron with an extremely low rate of secretion 


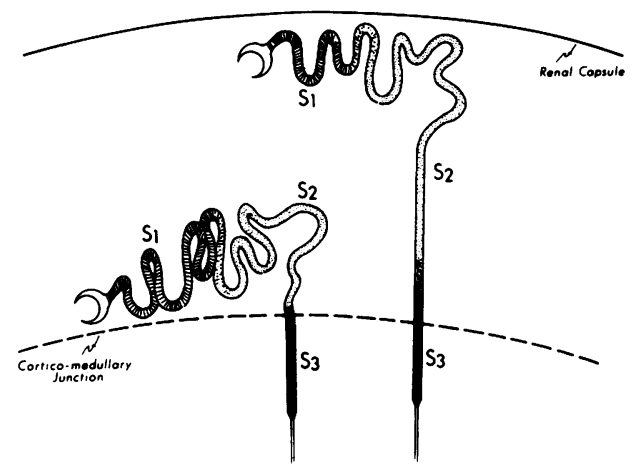

Figure 3 Schematic representation of the distribution of the $S_{1}, S_{2}$, and $S_{3}$ segments defined by cell type in $S F$ and JM proximal tubules. Transition regions are indicated between $S_{1}$ and $S_{2}$ of both SF and JM proximal tubules and between $S_{2}$ and $S_{3}$ of $S F$ tubules. See text for explanation.

( $28 \mathrm{fmol} / \mathrm{mm}$ per $\mathrm{min}$ ). Net secretory rates of $S_{1}$ and $S_{3}$ segments of JM nephrons did not differ statistically $(P>0.05)$ when this single tubule was excluded from the calculations. Importantly, net PAH secretion was also found to be comparable in morphologically identical segments of SF and JM nephrons. Thus, with respect to $\mathrm{PAH}$ secretion, evidence of functional heterogeneity between SF and JM nephrons was not observed when comparisons were made between segments whose cellular morphology was identical.

In an attempt to exclude the possibility that low net PAH secretion by $S_{3}$ segments was due to incubation of the segments in a bath which was less hypertonic than the interstitial fluid which normally bathes the tubules in vivo, six $S_{3}$ segments were studied in

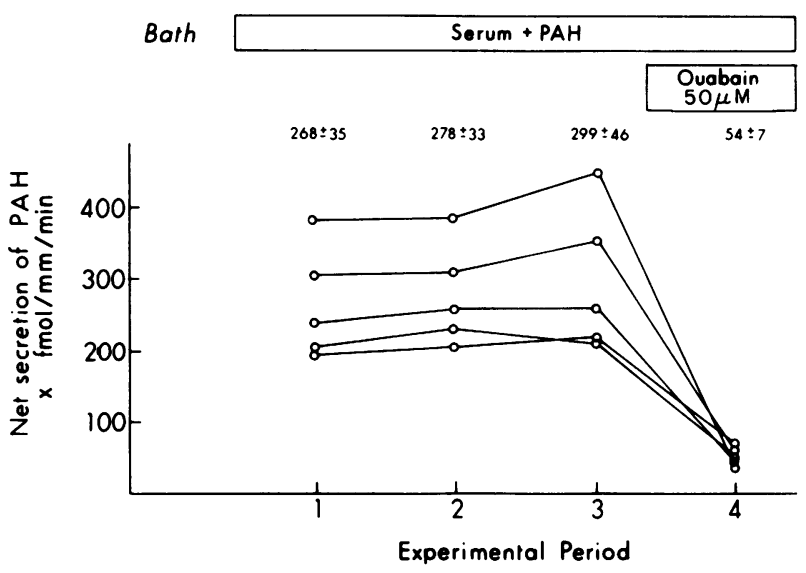

Figure 4 PAH secretion in $S_{1}$ segments of $\mathrm{SF}$ proximal tubules before (periods 1-3) and after (period 4) ouabain.

which the osmolality of the perfusate and bath was increased to $350-360 \mathrm{mosmol} / \mathrm{kg} \mathrm{H}_{2} \mathrm{O}$ by the addition of $\mathrm{NaCl}$. The mean net PAH secretion in this group of six $\mathrm{S}_{3}$ segments obtained randomly from SF $(n=3)$ and JM $(n=3)$ proximal tubules was $281 \pm \mathrm{SE} 42 \mathrm{fmol} / \mathrm{mm}$ per min. This value was not statistically different from a mean value of $253 \pm \mathrm{SE} 50 \mathrm{fmol} / \mathrm{mm}$ per min obtained by pooling the data obtained from the JM and SF $S_{3}$ segments that were bathed and perfused in an isosmotic ultrafiltrate of rabbit serum.

\section{DISCUSSION}

The results of the present study provide a detailed structural-functional analysis of PAH secretion in prox-

TABLE II

PAH Secretion in Individual Segments of SF and JM Proximal Tubules

\begin{tabular}{|c|c|c|c|c|c|c|c|c|c|c|c|c|}
\hline & \multicolumn{4}{|c|}{$S_{1}$ segment } & \multicolumn{4}{|c|}{$S_{2}$ segment } & \multicolumn{4}{|c|}{$\mathrm{S}_{3}$ segment } \\
\hline & \multicolumn{2}{|c|}{ SF } & \multicolumn{2}{|c|}{$\mathrm{JM}$} & \multicolumn{2}{|c|}{ SF } & \multicolumn{2}{|c|}{$\mathrm{JM}$} & \multicolumn{2}{|c|}{ SF } & \multicolumn{2}{|c|}{$\mathrm{JM}$} \\
\hline & Normal & Ouabain & Normal & Ouabain & Normal & Ouabain & Normal & Ouabain & Normal & Ouabain & Normal & Ouabain \\
\hline \multirow{9}{*}{$\begin{array}{l}\text { Net PAH secretion, } \\
\text { fmol } / \mathrm{mm} \\
\text { tubule } / \mathrm{min}\end{array}$} & $(n=15)^{*}$ & $(n=5)$ & $(n=15)$ & $(n=5)$ & $(n=27)$ & $(n=6)$ & $(n=12)$ & $(n=4)$ & $(n=15)$ & $(n=3)$ & $(n=15)$ & $(n=5)$ \\
\hline & 210 & 74 & 433 & 61 & $1,326 \rrbracket$ & 76 & 1,772 & 122 & 311 & - & 189 & 57 \\
\hline & 406 & 38 & 493 & 75 & $1,910 \ddagger$ & 120 & 1,180 & 126 & 181 & - & 243 & 64 \\
\hline & 247 & 36 & 401 & 36 & $1,286 \sharp$ & 118 & 1,211 & 121 & 639 & 67 & 28 & 14 \\
\hline & 331 & 64 & 231 & 59 & 1,507 & - & 1,402 & 133 & 167 & 60 & 266 & 82 \\
\hline & 216 & 56 & 206 & 77 & 1,268 & 75 & & & 292 & 98 & 216 & 50 \\
\hline & & & & & 1,406 & 70 & & & & & & \\
\hline & & & & & 948 & - & & & & & & \\
\hline & & & & & 1,624 & 122 & & & & & & \\
\hline Mean \pm SE & $281 \pm 21 \S$ & $54 \pm 7$ & $353 \pm 31 \S$ & $62 \pm 7$ & $1,508 \pm 104^{\prime \prime}$ & $97 \pm 10$ & $1,391 \pm 72^{\prime \prime}$ & $126 \pm 3$ & $318 \pm 469$ & $75 \pm 12$ & $188 \pm 239$ & $53 \pm 11$ \\
\hline $\begin{array}{l}\text { Tubule length,** } \\
\mathrm{mm} \pm \mathrm{SE}\end{array}$ & \multicolumn{2}{|c|}{$1.23 \pm 0.11$} & \multicolumn{2}{|c|}{$1.13 \pm 0.14$} & \multicolumn{2}{|c|}{$1.06 \pm 0.11$} & \multicolumn{2}{|c|}{$1.22 \pm 0.09$} & \multicolumn{2}{|c|}{$1.23 \pm 0.11$} & \multicolumn{2}{|c|}{$1.13 \pm 0.12$} \\
\hline
\end{tabular}

* No. of periods of observation.

\ Late PC just preceding the descending portion of the tubule.

$\$ S_{1}$ segment, $\mathrm{SF}$ vs. JM $-P>0.15$.

" $\mathrm{S}_{2}$ segment, $\mathrm{SF}$ vs. JM $-P>0.45$.

If $S_{3}$ segment, $S F$ vs. JM $-P>0.10$.

** Length of perfused segment. 


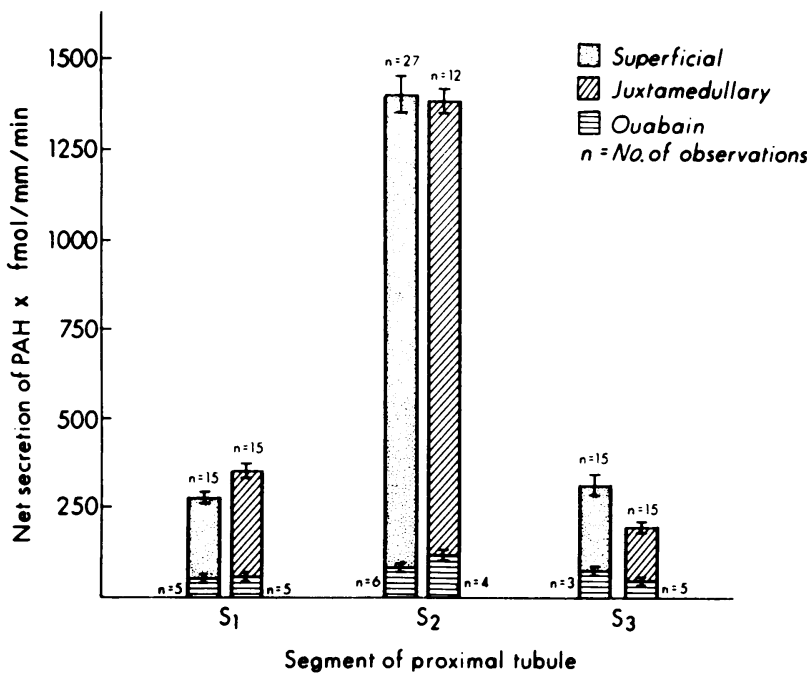

Figure 5 Summary of PAH secretion in all three segments of $\mathrm{SF}$ and JM proximal tubules. Maximal secretory rates were observed in $S_{2}$ segments of both nephron populations.

imal tubules of SF and JM nephrons of the rabbit kidney. On the basis of cellular morphology alone, it was possible to subdivide the proximal tubule into three distinct segments $\left(S_{1}, S_{2}\right.$, and $\left.S_{3}\right)$ and to establish that the segmentation was similar in both $\mathrm{SF}$ and JM proximal tubules. PAH secretion was found to be maximal in the $S_{2}$ segment and similar secretory patterns were noted in both SF and JM proximal tubules.

Although previous morphological studies in mouse (5), rat (4), and the rhesus monkey (6) have provided evidence of cellular segmentation within the proximal tubule, the presence of at least three distinct segments has not been documented previously in the rabbit proximal tubule. In addition, the present observations provide evidence in the mammalian kidney that both the SF and JM proximal tubules are composed of at least three anatomic segments. Although the division of the proximal tubule into the $P C$ and the $P R$ on the basis of external gross and macroscopic anatomic features has been found convenient for purposes of examining a large variety of functional events in this region of the nephron, examination of Fig. 3 reveals that in many instances two cellularly distinct segments may be included in a particular functional analysis when the proximal tubule is subdivided in this manner. For example, most micropuncture studies are commonly conducted along the initial $60 \%$ of the proximal tubule and, in many instances, functional data are undoubtedly obtained from either the $S_{1}$ or $S_{2}$ segments, or both. It is likely that punctures of "late" SF proximal convoluted tubules most often involve the $S_{2}$ segment. Indirect evidence in support of this conclusion can be mustered by comparing the results of recent micropuncture observations in the rabbit with certain mor- phological data in the present study. Chonko et al. (13) measured the length of the proximal tubule accessible to micropuncture in $12 \mathrm{SF}$ nephrons obtained from rabbits weighing $2-2.8 \mathrm{~kg}$ by the latex cast method. Their value of $5.40 \mathrm{~mm}$ is essentially identical to that of $5.30 \pm \mathrm{SE} 0.10 \mathrm{~mm}$ for the $\mathrm{PC}$ of $\mathrm{SF}$ proximal tubules of young animals those weight averaged $2.1 \mathrm{~kg}$ in the present study. If, as is generally assumed, the portion of the proximal tubule that is accessible to micropuncture represents $\sim 60 \%$ of its entire length, it could be estimated that the length of the entire proximal tubule would $\sim 9 \mathrm{~mm}$. This latter value compares quite favorably with our measured value of $8.69 \pm \mathrm{SE} 0.14 \mathrm{~mm}$ in SF proximal tubules of young animals. Thus, at least in the rabbit, the puncture of "late" SF proximal convoluted tubules would, in all likelihood, fall well within an $S_{2}$ segment as defined in the present study. The problem of physiological sampling from morphologically heterogeneous tubule segments may also exist in studies which involve the use of isolated perfused tubules. This issue will be addressed in greater detail in subsequent sections.

The results of the PAH secretory studies reveal the existence of functional heterogeneity along the length of the proximal tubule in both SF and JM nephrons. The highest rate of PAH secretion was noted in the $\mathrm{S}_{2}$ segment of both $\mathrm{SF}$ and JM nephrons, whereas the $S_{1}$ and $S_{3}$ segments exhibited a lower capacity for PAH secretion. At first glance these findings appear to be at variance with earlier measurements of $\mathrm{PAH}$ secretion in the isolated perfused rabbit proximal tubule (1). The mean net PAH secretion by the PC was $2.35 \pm 38$ fmol $/ \mathrm{mm}$ per min, a value very similar to that obtained in $S_{1}$ segments of $S F$ and JM proximal tubules in the present study. The results suggest that Tune et al. (1) were studying relatively early regions of the $\mathrm{PC}$ which would correspond to the $S_{1}$ segment. These same workers reported a mean value for net PAH secretion of $869 \pm 52 \mathrm{fmol} / \mathrm{mm}$ per min for the straight portion or PR of proximal tubules whose nephron of origin, that is, SF or JM, was not identified. Based on our own findings, it is readily apparent that if $\mathrm{PAH}$ secretion is examined in the $\mathrm{PR}$ of proximal tubules from $\mathrm{SF}$ nephrons without regard to the cellular composition of the tubule under study, segments of high $\left(S_{2}\right)$ as well as low $\left(\mathrm{S}_{3}\right)$ PAH secretion may be included in the preparation. Their value of $869 \pm 52 \mathrm{fmol} / \mathrm{mm}$ per min obtained from straight segments is approximately midway between the mean net PAH secretory rates obtained in the $S_{2}$ and $S_{3}$ segments in the present investigation and may reflect inclusion of tubules representing a mixture of the two segments. This potential pitfall was intentionally avoided in the present study by the selection of short tubule segments of uniform cell type from well within the boundries of the three segments as defined initially via light and electron 
microscopy. Thus, we conclude that it is the $S_{2}$ segment, and not the PR of the proximal tubule, where maximum PAH secretion is principally located.

The present investigation represents one of the few studies in which a functional comparison has been made between morphologically identical segments of SF and JM nephrons. In the present study SF and JM proximal tubules were divided initially into specific segments carefully defined according to characteristic morphologic features and then, on the basis of these features, examined functionally to determine whether heterogeneity did exist between comparable regions of different nephron populations for a particular physiologic event. Although striking differences in net PAH secretion were observed from one cellular segment to another along the proximal tubule, evidence of functional heterogeneity between different nephron populations was not found. It should be noted, however, that an apparent difference in PAH secretion between straight segments of SF and JM proximal tubules might well have been observed if the usual gross anatomic criteria that have been employed in many studies had been followed in the present experiments. This is because, of course, virtually all of the PR of JM proximal tubules is composed of $S_{3}$ cells, whereas much of the $\mathrm{PR}$ of $\mathrm{SF}$ proximal tubules is cortical in location and composed principally of $S_{2}$ cells. Thus, any functional comparison between the PR of JM and SF proximal tubules will most likely include a comparison of JM $\mathrm{S}_{3}$ segments with either SF $\mathrm{S}_{2}$ segments, if the latter are dissected exclusively from the outer cortex, or a combination of SF $\mathrm{S}_{2}$ and $\mathrm{SF}_{3}$ segments if both cortical and outer medullary SF straight segments are studied.

Although our studies failed to reveal the presence of functional heterogeneity between different nephron populations, other investigators employing the isolated perfused tubule technique have described apparent functional differences between SF and JM proximal tubules. Kawamura et al. (14) examined the sodium and chloride permeabilities of SF and JM straight segments, the former derived from both the cortex and the outer medulla. The results of both electrophysiological and isotopic estimates of chloride to sodium permeability suggested that $\mathrm{SF}$ straight segments were approximately two times more permeant to chloride than to sodium. The converse was true of JM straight segments. In a later study, Jacobson and Kokko (15) compared the relative chloride and sodium permeabilities in SF and JM proximal convoluted tubules by electrophysiological techniques only. Sodium permeability was found to exceed chloride permeability in the early portion of the $\mathrm{PC}$ (probably the $\mathrm{S}_{1}$ segment) in both SF and JM proximal tubules. However, the relative sodium permeability continued to exceed the chloride permeability throughout the remainder of the JM proximal convoluted tubule (probably corresponding to the late $S_{1}$ and $S_{2}$ segments), whereas the converse was true in the more distal regions of the SF proximal convoluted tubule $\left(S_{2}\right.$ segment). In contrast to the results obtained for PAH secretion, these data suggest that functional heterogeneity between anatomically similar segments of different nephron populations exists for chloride and sodium permeabilities along the proximal tubule.

Recently, Warnock and Burg (16) reported a difference in permeability and transport rate of $\mathrm{CO}_{2}$ between isolated perfused proximal straight tubules of SF and JM nephrons of the rabbit. Although a definite attempt was made by these investigators to study proximal straight tubules from two distinct nephron populations, it is not possible to ascertain from the published account of the methodology that was employed whether morphologically identical segments based on the cellular composition were actually examined.

\section{ACKNOWLEDGMENTS}

The authors gratefully acknowledge the technical assistance of Mrs. Helen Parks and Mrs. Kathy Blake and the secretarial contribution of Mrs. Greta Owens. Mr. William Boyarsky and Mrs. Jessie Calder prepared the illustrative material.

This work was supported in part by U. S. Public Health Service grants AM 13845, HL 05848, and AM 17195.

\section{REFERENCES}

1. Tune, B. M., M. B. Burg, and C. S. Patlak. 1969. Characteristics of $p$-aminohippurate transport in proximal renal tubules. Am. J. Physiol. 217: 1057-1063.

2. Suzuki, T. 1912. Zur Morphologie der Nierensekretion unter physiologischen und pathologischen Bedingungen. Fischer, Jena.

3. Sjöstrand, F. S. 1944. Uber die Eigenfluoreszenz tierischer Gewebe mit besondrer Berucksichtigung der Saugetierniere. Acta. Anat. 1(Suppl. 1): 1-163.

4. Maunsbach, A. B. 1966. Observations on the segmentation of the proximal tubule in the rat kidney. Comparison of results from phase contrast, fluorescence and electron microscopy. J. Ultrastruct. Res. 16: 239-258.

5. Rhodin, J. 1962. Electron microscopy of the kidney. In Renal Disease. D. A. K. Black, editor. Blackwell Scientific Publications, Ltd., Oxford. 117.

6. Tisher, C. C., S. Rosen, and G. B. Osborne. 1969. Ultrastructure of the proximal tubule of the rhesus monkey kidney. Am. J. Pathol. 56: 469-517.

7. Burg, M. B., J. Grantham, M. Abramow, and J. Orloff. 1966. Preparation and study of fragments of single rabbit nephrons. Am. J. Physiol. 210: 1293-1298.

8. Allen, F., and C. C. Tisher. 1976. Morphology of the ascending thick limb of Henle. Kidney Int. 9: 8-22.

9. Woodhall, P. B., and C. C. Tisher. 1973. Response of the distal tubule and cortical collecting duct to vasopressin in the rat. J. Clin. Invest. 52: 3095-3108.

10. Tisher, C. C., and J. R. Clapp. 1972. Intraluminal latex injection: An aid to the histological identification of renal tubules. Kidney Int. 2: 54-56. 
11. Sperber, I. 1944. Studies on the mammalian kidney. Zool. Bidr. Upps. 22: 249-431.

12. Schønheyder, H. C., and A. B. Maunsbach. 1975. Ultrastructure of a specialized neck region in the rabbit nephron. Kidney Int. 7: 145-153.

13. Chonko, A. M., R. W. Osgood, A. E. Nickel, T. F. Ferris, and J. H. Stein. 1975. The measurement of nephron filtration rate and absolute reabsorption in the proximal tubule of the rabbit kidney. J. Clin. Invest. 56: 232-235.
14. Kawamura, S., M. Imai, D. W. Seldin, and J. P. Kokko. 1975. Characteristics of salt and water transport in superficial and juxtamedullary straight segments of proximal tubules. J. Clin. Invest. 55: 1269-1277.

15. Jacobson, H. R., and J. P. Kokko. 1976. Intrinsic differences in various segments of the proximal convoluted tubule. J. Clin. Invest. 57: 818-825.

16. Warnock, D. G., and M. B. Burg. 1977. Urinary acidification: $\mathrm{CO}_{2}$ transport by the rabbit proximal straight tubule. Am. J. Physiol. 232: F20-F25. 